\title{
¿Conocemos las características epidemiológicas, clínicas, estrategias de tratamientos y el pronóstico de los pacientes con síndrome coronario agudo?
}

\section{Do we know the epidemiological, clinical, treatment strategies and prognosis of patients with acute coronary syndrome?}

Definitivamente la enfermedad cardiovascular en Colombia y en el mundo es la causa líder de morbilidad y mortalidad, el conocimiento de las características clínicas y de intervención en la manera de registros nos brindaría una información importantísima de la forma como se hace la presentación clínica de los pacientes con síndrome coronario agudo y de los resultados de la intervención farmacológica y no farmacológica, permitiéndonos hacer modificaciones que redunden en beneficio de nuestros pacientes con esta condición, y permitiéndonos conocer el nivel de intervención en nuestro país de estrategias soportadas por la evidencia y de la aplicación de las guías de practica clínica.

En el trabajo de Chavarriaga y cols. (1), de las características epidemiológicas, clínicas, tratamiento y pronóstico de los pacientes con diagnóstico de síndrome coronario agudo en unidad especializada, un estudio epidemiológico, observacional, descriptivo, longitudinal, retrospectivo tipo serie de casos, evaluados en un periodo de tres meses, a quienes se les realizó tratamiento en unidad especializada de manejo de pacientes con dolor torácico y hallazgos clínicos y de laboratorio de síndrome coronario agudo, y se les realizó un seguimiento de su condición a los seis meses, por varios medios, ya sea contacto directo con evaluación por consulta externa, evaluación de sus historias clínicas, o contacto telefónico con el fin de obtener información sobre evolución clínica estado funcional, complicaciones, reintervenciones, hospitalizaciones o muerte, adicionalmente a la adherencia a los tratamiento instaurados al alta.

Se siguieron un total de 154 pacientes con distribución de características de SCA así:
$30 \%$ de los pacientes con diagnóstico de angina inestable, $37 \%$ con diagnóstico de infarto de miocardio con elevación del segmento ST, y $33 \%$ con diagnóstico de infarto sin elevación del segmento ST. Ésto como bien lo comentan los investigadores de este estudio, se parece bien a las estadísticas internacionales, como es el caso del registro GRACE que mostró que el porcentaje de pacientes con IAM con elevación del ST de un 34\%, la angina inestable de $29 \%$ y el infarto sin elevación del segmento ST de $33 \%$ (2).

El promedio de edad fue de $62 \pm 13$ años, con hombres del $54 \%$, siendo la población del registro GRACE ligeramente mayor entre 65 y 70 años con un predominio del sexo masculino de $64-70 \%$ (2).

Los factores de riesgo asociados en esta cohorte de pacientes fueron la hipertensión arterial en $66 \%$, la dislipidemia en $23 \%$, la diabetes mellitus $18 \%$, la obesidad $5,1 \%$ y el tabaquismo 52\%. En el registro GRACE, la hipertensión arterial estuvo presente en el $58 \%$ de los pacientes, menor que en la cohorte de este estudio, dislipidemia se encontró en $42 \%$ mucho más elevada en este grupo que en la cohorte del estudio al igual que la diabetes, la cual se encontró en el registro GRACE en $24 \%$ también muy superior a la encontrada en este trabajo (2). En el caso del tabaquismo en el registro GRACE, éste estuvo presente en $58 \%$ de los pacientes también superior comparativamente con el registro de Chavarriaga y cols.

El número total de estudios de angiografía coronaria realizados en los pacientes de esta cohorte fueron 129 (83\%), y de los pacientes con IAMST, sólo $28 \%$ recibieron reperfusión primaria, a diferencia del registro GRACE,
Ver artículo: página 21

Dr. Efraín Alonso Gómez López: Especialista en Medicina Interna, Cardiología y Medicina Crítica y Cuidados Intensivos. Jefe de la Unidad de Cuidados Intensivos Coronarios de la Clínica Shaio. Presidente de la Sociedad Colombiana de Cardiología y Cirugía Cardiovascular. Bogotá, D.C. (Colombia). E-mail: eag197@gmail.com 
que mostró que los pacientes con IAMST quienes tuvieron intervención percutánea primaria 610 (48\%), adicionalmente 13\% (165) tuvieron angioplastia de rescate por trombolisis fallida; en total la estrategia de reperfusión terapia lítica o angioplastia primaria fue usada en el registro GRACE en $62 \%$ de estos pacientes (2), lo cual es marcadamente superior a la del registro de Chavarriaga y cols.

La mortalidad intra hospitalaria en el estudio de Chavarriaga y cols., de todos los pacientes con síndrome coronario agudo fue de $7 \%$ y a los seis meses fue de $12 \%$ con una pérdida del seguimiento de $19 \%$ que podría influir en el verdadero resultado de mortalidad a los seis meses y podría aumentar la diferencia con los resultados del GRACE que a seis meses mostró una mortalidad de $4.7 \%$, si bien es cierto la mortalidad intrahospitalaria del registro GRACE fue en promedio del 7.5\% con mayor mortalidad de los pacientes con IAMST (7\%), de 5\% para el IAMNST y de $3 \%$ para los pacientes con angina inestable, el hecho de que el registro de Chavarriaga tuviese un grupo significativo de bajo riesgo (30.6\%), que pudo haber influido para que los resultados de mortalidad no fueran mayores.

Adicionalmente a todo ello se encontró que la adherencia al tratamiento fue pobre durante el seguimiento (entre 54 y $86 \%$ ), lo cual seguramente tuvo un impacto significativo en la contribución de la mayor mortalidad a seis meses.

Definitivamente este importante esfuerzo de evaluar una cohorte de pacientes con síndrome coronario agudo y su posterior seguimiento a seis meses nos enseña claramente que la falta de estrategias de reperfusión fibrinolítica o percutáneas especialmente en este registro por presentación fuera de ventana terapéutica, tienen repercusiones en la mortalidad intrahospitalaria, y sumado a la pobre adherencia al tratamiento de medicaciones soportadas en la evidencia de beneficio clínico, trajeron resultados con mayor mortalidad a seis meses de seguimiento en esta cohorte cuando se comparan con los resultados del registro GRACE (2).
Felicito al grupo de Chavarriaga y colaboradores por este importante esfuerzo de evaluar los resultados en esta cohorte, que nos muestra la necesidad de realizar un registro nacional de síndrome coronario agudo, que involucre además instituciones de mediano nivel de complejidad para reconocer la verdadera realidad del comportamiento en nuestro país de los pacientes quienes se presentan con un síndrome coronario agudo. En la Sociedad Colombiana de Cardiología hemos trabajado este año para la consecución de la base de datos, para el inicio del registro nacional de síndrome coronario agudo, la cual ya tenemos en nuestro poder y estamos ajustando detalles de logística para iniciar un ambicioso proyecto de registro de pacientes de síndrome coronario agudo en nuestro país; en este esfuerzo debemos trabajar mancomunadamente todos los estamentos involucrados en la atención en salud de nuestros pacientes con síndrome coronario agudo.

Sin duda alguna, el registro clínico nos dará respuesta más claramente sobre la realidad de la atención y los resultados de las intervenciones de los pacientes con síndrome coronario agudo en Colombia. Esta cohorte de Chavarriaga y cols. nos muestra una parte de la realidad de nuestro país, pero es la cara de una institución de atención de tercer nivel y no tenemos claridad sobre cómo sería una situación similar en instituciones de menor nivel de complejidad que tengan que enfrentar pacientes con síndrome coronario agudo.

En conclusión, en nuestro país no conocemos las características epidemiológicas, clínicas, estrategias de tratamientos, ni el pronóstico de los pacientes con síndrome coronario agudo.

\section{Referencias}

1. Chavarriaga JC, Beltrán J, Senior JM, Fernández A, Rodríguez A, Toro JM. Características epidemiológicas, clínicas, tratamiento y pronóstico de los pacientes con diagnóstico de síndrome coronario agudo en unidad especializada. Acta Med Colomb 2014; 39: 21-28.

2. Philippe Gabriel Steg, MD, Robert J. Goldberg, PhD, Joel M. Gore, MD. Baseline Characteristics, Management Practices, and In-Hospital Outcomes of Patients Hospitalized With Acute Coronary Syndromes in the Global Registry of Acute Coronary Events (GRACE). Am J Cardiol 2002; 90: 358-363 